\title{
La responsabilidad social en Latinoamérica: Una perspectiva desde la Ingeniería Industrial
}

\author{
Salazar Roldán Marcelo Nicolás \\ ORCID: https://orcid.org/0000-0003-4818- \\ $370 \mathrm{X}$ \\ marcelo.salazar.roldan@udla.edu.ec \\ Universidad de las Américas \\ Quito-Ecuador
}

Recibido (09/11/20), Aceptado (18/11/20)

Resumen: En este trabajo de investigación se va abordará temas de carácter empresarial y relacionados con su responsabilidad social. Así mismo, se realizará una comparación entre continentes para conocer los diferentes aspectos según la localización de las compañías y sus participantes. De igual manera, se abordará un análisis mencionando diferentes definiciones con respecto a este tema y el rol de un ingeniero industrial en estos aspectos. Después de argumentar y sobrellevar todos los temas acerca de empresas socialmente responsables, se presentará un ejemplo que cumpla todos los puntos establecidos previamente. Antes de finalizar, desde el punto de vista social se ilustrará posibles escenarios en los que podría ser factible la implementación de la responsabilidad social empresarial y finalmente se llegaría a establecer los factores que influyen en las organizaciones o empresas para que incumplan la responsabilidad social.

Palabras Clave: responsabilidad social, ingeniero industrial, organizaciones.

\section{Social responsibility in Latin America: A perspective from Industrial Engineering}

\begin{abstract}
This research work will address business issues and related to social responsibility. Likewise, a comparison will be made between continents to know the different aspects according to the location of the companies and their participants. In the same way, an analysis will be approached mentioning different definitions regarding this topic and the role of an industrial engineer in these aspects. After arguing and overcoming all the issues about socially responsible companies, an example will be presented that meets all the previously established points. Before concluding, from the social point of view, possible scenarios in which the implementation of corporate social responsibility could be feasible will be illustrated and finally the factors that influence organizations or companies to fail to complywith social responsibility will be established
\end{abstract}

Keywords: social responsibility, industrial engineer, organizations. 


\section{I.INTRODUCCIÓN}

Si se desea obtener un desarrollo óptimo dentro de un producto o servicio, la empresa está en la obligación de diseñar estrategias que promuevan la eficiencia y mejora continua en sus proyectos con el objetivo de generar expectativa en el cliente final. Es importante resaltar la importancia de los valores que debe poseer una organización, entre estos la transparencia se ha convertido y ha representado un papel fundamental para el buen vivir dentro de las empresas socialmente responsable. Los beneficios no solamente son unidireccionales sino también proveen de ellos a los "Stakeholders" de la misma.

Por lo general, la terminología "Bienestar Social” no se la relaciona con el ámbito empresarial, sin embargo, estos dos aspectos van llevados de la mano, ya que se debe buscar el bienestar social antes que el beneficio personal. Cuando una empresa entiende esta premisa y la aplica, se llega a consolidar una ventaja competitiva productivamente y un mejor ambiente laboral. El presente artículo pretende dar una contribución a la sociedad y a las empresas realizando una definición y un análisis que servirá para los cimientos de éxito para que una empresa pueda emprender preocupándose con la responsabilidad social empresarial. Debido a que hoy en día, este tipo de estrategia ayuda a las organizaciones a competir tanto a nivel nacional como internacional.

\section{DESARROLLO}

Con el objetivo de facilitar la comprensión del tema, se comenzará describiendo el concepto de Responsabilidad Social o mejor conocido por sus siglas "RSE". La cual se establece como, la condición y el discernimiento que tiene como finalidad el equilibrio entre desarrollar de forma adecuada la sociedad, crear sustentabilidad en todo tipo de entorno y por ende tiene que ser viable económicamente. Una Empresa Socialmente responsable siempre integrará principalmente los aspectos sociales y medio ambientales. Así mismo, integra el aspecto monetario, cabe recalcar que a pesar de que el aspecto económico es de suma importancia en este artículo es considerado intrínseco debido al enfoque de prioridad asignado hacia cualquier sistema de administración empresarial.

Socialmente hablando, una organización debe considerar notablemente aspectos de mejora, desarrollo e importancia de factores que tengan relación directa con el recurso humano de la institución, tanto a nivel interno como externo de la empresa. La transparencia ha sido una de las cualidades que en los últimos tiempos ha estado entrando fuerte y representativamente relacionando a los clientes, socios, gobierno, consumidores, proveedores medio ambiente y comunidades. Al dar la importancia que se merece esta peculiaridad como es la transparencia en las empresas, se llega a consolidar una estrategia de negocios representativa que generará competitividad, sostenibilidad y rentabilidad.

La empresa se ve en la obligación de tener compromiso hacia la sociedad para la correcta contribución hacia el desarrollo y el bienestar común. De esta manera, la calidad de vida de la comunidad va a mejorar notablemente como así también los lazos generados entre empresa y participantes se va a entrelazar de mejor manera generar un compromiso y mayores oportunidades en el futuro. El RSE tiene como visión general desarrollar el máximo nivel de utilidad siempre y cuando se respeten las normativas regulatorias sociales y ambientales. Es importante señalar que no solamente se debe cumplir y respetar dichas normativas sino también se debe tomar en cuenta la ética y valores morales entre diferentes grupos de interés de la empresa sea de manufactura o servicio. [1]

La siguiente comparación se efectuó de una manera global por medio de una recolección de datos en los diferentes continentes. Lo cual dio como resultado que en Latinoamérica existen diferentes organizaciones las cuales están dedicadas a la regulación, establecimiento y creación de normativas destinadas a la RSE, entre las principales organizaciones se encuentran:

-ACNUOH: Vela por los derechos humanos

-OCDE: Promueve el bienestar económico y social

-OIT: Establece normas laborales

Es común en Latinoamérica, mediante reportes globales periódicos lograr conseguir certificaciones que ayudan al RSE [2].

Por otro lado, en Europa, el ente regulador está establecido por medio de la Unión Europea la cual señala que debe existir dentro de las organizaciones una política comercial y política de desarrollo sostenible para garantizar un desarrollo económico, establecido por los siguientes aspectos:

-Justicia Social 
- Respeto a los derechos humanos

-Altos estándares laborales y ambientales

La Unión Europea beneficia a las empresas por medio acuerdos comerciales en el continente e incentivos especiales hacia las organizaciones que cumplan y promuevan esta buena práctica [3].

En los últimos años Asia y África han conseguido una responsabilidad social aceptable sobre todo en lo que respecta a los derechos humanos, pero también se redujo la mortalidad de menores de edad.

Por un lado, Asia, mediante sistemas de gestión y políticas con sus "Stakeholders" y por otro lado África maneja cuatro aspectos los cuales son:

- Obligar

- Facilitar

- Colaborar

-Promocionar

Parecería que los Estados Unidos, al ser un país del primer mundo sabe sobrellevar de manera óptima el tema de la responsabilidad social, sin embargo, ellos camuflan y confunden el término, responsabilidad empresarial con responsabilidad social. Pues tienen un enfoque netamente remunerado sea privado o estatal, afirmando que los puntos mencionados anteriormente junto con la certificación y capacitación son más que suficientes [4] [5] [6].

Para poder entrelazar la Responsabilidad Social Empresarial con la diciplina de la Ingeniería Industrial, se debe establecer el concepto de a que se refiere la misma. Dicho esto, se puede afirmar que al hablar de la Ingeniería Industrial se hace referencia al análisis, comprensión, diseño e interpretación para el control de sistemas de producción en una organización, para a continuación gestionar e implementar mejoras, estrategias y metodologías con el fin de conseguir un rendimiento superior en los procesos y procedimientos de la empresa, con el objetivo de alcanzar la anhelada eficacia o eficiencia organizacional. Por esta razón, un ingeniero industrial cumple un papel muy importante en cualquier tipo de organización sin importar el ámbito que se dedique la empresa, sea manufactura, servicios o comercialización. Debido a que, ayuda a cumplir el principal objetivo de cualquier institución, mediante la sostenibilidad y rentabilidad de esta.

Aclarado este tema, se procederá a efectuar la relación entre la ingeniería industrial y la responsabilidad social empresarial. Al ser una de las principales cualidades de este tipo de profesionales con respecto a la focalización de aspectos de planeación y mejora de toda la parte organizacional de la empresa, comenzando desde lo administrativo, operacional, metodológico e incluso hasta legislativo. Todo esto se lleva a cabo por medio del control y estandarización de los procesos y procedimientos, lo cual ayuda a tener un panorama más claro y una ilustración representativa del funcionamiento y estructura de la empresa. Es decir, un ingeniero industrial está encargado de controlar la calidad de un producto o servicio sin afectar el medio ambiente o a la sociedad, también de controlar el uso y manipulación adecuada de desechos y sustancias. Finalmente, es trabajo de este profesional encaminare de manera íntegra cada mejora, proceso y acción de negocio que vaya a tomar la empresa.

Como ejemplo de una Empresa Socialmente Responsable se seleccionó a una de las organizaciones más representativas a nivel mundial con respecto a la elaboración y comercialización de bebidas gasificadas. "Coca-Cola Company" se encuentra dentro de las cinco mejores empresas con respecto a las buenas prácticas de responsabilidad social, ya que posee altos niveles de contribución caritativa. La empresa se establece por tres formas del bienestar, las cuales son muy importantes:

-Individual

-Social

-Ambiental

Hoy en día, como respaldo de eventos y acciones que impulsen un desarrollo para el entorno que sea sustentable y optimista para el futuro. Un compromiso el cual efectuó la empresa es devolver a la naturaleza la mayor cantidad de agua que emplea sus procesos de producción, mediante un programa de reabastecimiento, reciclado y reducción [7].

Existen 8 principales factores, los cuales por medio de su influencia promueven al incumplimiento de la Res- 
ponsabilidad Social en las organizaciones. Estos factores son los siguientes:

- Pensamiento limitado de aplicación en organizaciones de carácter grande y multinacional

-Creencia de limitación de responsabilidades en empresas que en sus prácticas están involucradas directamente al deterioro de aspectos ambientales o sociales

-Pensamiento relacionado y limitado al RSE con respecto a donaciones

-Pensamiento conformista legislativo

- Falta de compromiso de la alta gerencia con respecto a la implantación de un proyecto de RSE

- Creencia de que basta con una sola implantación de RSE

- Pensamiento corporativo referente al bienestar interno social relacionado al cumplimiento de salarios y ambiente laboral adecuado

-Por lo general, las Pymes carecen de conocimiento con respecto a la RSE

La empresa que va más acorde con respecto a tener una cultura socialmente responsable sería la empresa multinacional Telefónica S.A. Ya que su fundamentación está establecida por la "Visión del Grupo". Al tener como principio la mejorar la vida de las personas como eje principal posteriormente se podrá tener beneficios tanto a nivel de desarrollo empresarial como también en contribución a la mejora de los "Stakeholders" en donde se encuentre la compañía [8].

La vinculación con la comunidad que se tiene por parte de Telefónica es impresionante ya que se premia a personajes de comunidades limitadas y de escasos recursos, como también a deportistas y personas con discapacidades. De igual manera la iniciativa con proyectos y pantallas por medio del incentivo de disminuir el uso de energía y cuidado del medio ambiente sembrando árboles son uno de los pocos aspectos que maneja Telefónica ética y moralmente.

\section{III.CONCLUSIONES}

Finalmente, se puede concluir al final de esta tesis, que de a poco se está logrando ingresar la ideología de Responsabilidad Social Empresarial en las organizaciones. Es un proceso difícil, ya que en la mayoría de los casos no se le aplica sea por conflictos de interés o por falta de conocimiento, sin embargo, organizaciones y los estados están generando campañas constantemente con el objetivo de promover y desarrollar esta práctica laboral en la mayoría de las empresas grandes. Sin embargo, existe un déficit en las pequeñas y medianas empresas al no tener la voluntad necesaria para promover las buenas prácticas laborales. Así mismo, existe una variabilidad en las empresas grandes con respecto a la empatía con este aspecto, ya que en algunos casos simplemente se las aplica por ser de carácter legal mas no voluntario. La Responsabilidad Social empresarial acarrea grandes beneficios, debido a que el eje fundamental del funcionamiento de una empresa es el recurso humano que se posee y el ambiente que los rodea. Si se logra generar un buen vínculo entre la empresa y esos dos aspectos, se generaría una productividad eficiente en las organizaciones y satisfacción emocional con los integrantes tanto internos como externos de la compañía.

\section{REFERENCIAS}

[1]E. Castaño, «Papel del Ingeniero Industrial en la Responsabilidad Social Empresarial,» 25 Junio 2012. [En línea]. Available: https://prezi.com/3_ittsoghcjw/articulo-papel-del-ingeniero-industrial-en-la-responsabilidad-social-empresarial/.

[2]V. Antonio y P. V. Estrella, «Inter-American Development Bank,» 201 1. [En línea]. Available: https://publications.iadb.org/publications/spanish/document/La-responsabilidad-social-de-la-empresa-en-Am\%C3\%A9rica-Latina-Manual-de-Gesti\%C3\%B3n.pdf.

[3]E. B. Fraile, «Universidad Autónoma de Barcelona,» [En línea]. Available: https://www.tdx.cat/bitstream/ handle/10803/384844/ebf1de1.pdf? sequence=1\&isAllowed $=y$.

[4]H. A. Franco, «Políticas públicas e iniciativa privada en la responsabilidad social empresarial,» [En línea]. Available: https://core.ac.uk/download/pdf/19712906.pdf.

[5]« Plataforma Digital de Derecho, Ciencias Sociales y Humanidades,» [En línea]. Available: https://leyderecho.org/responsabilidad-social-corporativa-en-asia/.

[6]«Compromiso Empresarial,» 2 Septiembre 2005. [En línea]. Available: https://www.compromisoempresarial.com/rsc/2005/09/caracteristicas-de-la-responsabilidad-social-en-las-empresas-de-estados-unidos/. 
[7]E. S.A., «EXPANSIÓN,» 23 Diciembre 2014. [En línea]. Available: https://expansion.mx/negocios/2014/12/16/las-10-empresas-mas-responsables-en-mexico.

[8]M. P. López, «Escuela de Organización Industrial,» 03 Febrero 2012. [En línea]. Available: https://www.eoi. es/blogs/migmamad/responsabilidad-social-corporativa-de-telefonica-s-a/\#: :text=El\%20objetivo\%20de\%20 la\%20estrategia,las\%20comunidades\%20donde\%20Telef\%C3\%B3nica\%20opera..

\section{RESUMEN CURRICULAR}

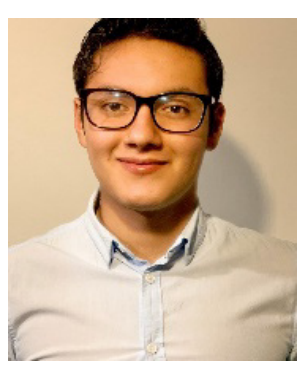

Marcelo Nicolás Salazar Roldán, ecuatoriano nacido el 03 de diciembre de 1999, en la ciudad de Quito Ecuador. Desempeñó sus labores profesionales en el área de producción en la empresa Dimabru Cia. Ltda. Estudiante de la carrera de Ingeniería Industrial en la Universidad de las Américas. Entre sus principios están el respeto, transparencia, empatía y puntualidad. Actualmente se encuentra realizando trabajos autónomos con respecto a la simulación y mejora continua de procesos 